\title{
Taguchi integrated Least Square Support Vector Machine an alternative to Artificial Neural Network analysis of electrochemical machining process
}

\author{
Kanhu Charan Nayak ${ }^{1}$, Rajesh Kumar Tripathy ${ }^{2}$ \\ ${ }^{I}$ Mechanical Engineering, National Institute of Technology, Rourkela, India \\ ${ }^{2}$ Biomedical Engineering, National Institute of Technology, Rourkela, India,
}

\begin{abstract}
The important performance parameter such as material removal rate(MRR) and surface roughness(SR) are influenced by various machining parameters namely voltage, feed rate of electrode and electrolyte flow rate in electrochemical machining process(ECM). In machining, the process of modelling and optimization are challenging tasks to qualify the requirements in order to produce high quality of products. There are a lot of modelling techniques that have been discovered by researchers. In this paper, the optimize settings of performance parameters; surface roughness and MRR are done by the Taguchi technique and the experimental result of MRR and SR was predicted by the Multi-layer Feed forward Neural network (MFNN) and Least square support vector machine (LSSVM). For Taguchi analysis three process parameters and two responses, MRR and SR were considered by L18 orthogonal array design and ANOVA result were performed. EN19 material used as the work piece for the experiment. After evaluating MFNN and LSSVM models, the best network found to be Least square support vector machine with RBF kernel. The mean square errors (MSE) between actual and predicted response obtained in both LSSVM model and MNFF model for the training and for testing datasets were concluded that LSSVM as more powerful machine learning tool and predict the MRR and SR successfully compared to other models. The performance of LSSVM is depend different kernel function that can separate the data from hyper plane for better prediction however we use Linear and RBF kernel. RBF kernel gives better prediction of MRR and SR with minimum MSE.
\end{abstract}

Keywords - ANOVA, EN-19 tool steel, LS-SVM, MFNN, Taguchi technique

\section{INTRODUCTION}

Electro chemical machining process is a non-conventional machining process to cut, high strength and high resistance materials into complex shapes. The working principle of ECM is just reverse the electro chemical planting(material is eroded from anode). High electrical current pass through the electrolyte medium which flows through between a tool (cathode) and work piece(anode). The mechanism of material removal is anodic dissolution according to the Faraday's laws of electrolysis. No residual stress form in ECM process and also no thermal damage due to low temperature during operation. This has widespread application in aerospace industry, automotive, forging die, and surgical component.

The Taguchi technique is a statistical method for designing high quality system, was developed by Taguchi. This method uses a special design of orthogonal array to study the entire parameter space with small number of experiment. Taguchi method is adopted to evaluate MRR and surface roughness. This method provides a systematic approach for conducting experimentation to determine optimum settings of design parameters.

We have seen that a variety of prediction models have been implemented in the literature that include time series models, regression models, adaptive neuro-fuzzy inference systems (ANFIS), artificial neural network (ANN) models and SVM models. In engineering the use of ANN has increased gradually. This is mainly because the effectiveness and smoothness of ANN modeling systems has improved a great deal in the engineering area. SVM have remarkable generalization performance and many more advantages over other methods, so SVM has attracted attention and gained extensive application. Not only LS-SVM has been used for classification in various areas of pattern recognition [17] but also it has handled regression problems successfully [18].LS-SVM has additional advantage as compared to SVM. In LS-SVM, a set of only linear equation (Linear programming) is solved which is much easier and computationally more simple. Here MFNN and LS-SVM models are used for prediction of material removal rate and surface roughness of EN19 material. After comparing the result obtained by both these models, LS-SVM gives better performance to minimize the MSE that of MFNN.

Muttamara A et la, presented relationship between ECM parameter and groove depth and groove ratio using Taguchi method [1]. Dubey A.K et la, presented the analysis of roundness of surface and out-of-roundness in ECM honing process[2]. Shan HS et la, showed parametric optimization of magnetic field assisted abrasive 
flow machining by taking different level of abrasive grain size, magnetic flux density, flow volume, extrusion pressure and work piece material using Taguchi method [3]. Asokan P. et la, Studied a practical method of multi-objective optimization in cutting parameters for ECM based on multiple regression models and multiple input single output ANN model [4]. Kuo Tsai et la., showed the ability of different neural networks models to predict the surface finish of work piece based on the effect of changing the electrode polarity in the EDM process. [5]. Shanget .Q.P et la, presented the effect of different process parameters on anode accuracy and developed an ANN model with back propagation as the learning algorithm to predict the anode accuracy in electrochemical machining with an uneven inter electrode gap [6]. Kozak, j.et la, showed the concept and prototype of a computer aided engineering system that can be used to solve different tasks of ECM, such as: tool-electrode design, selection of optimal input machining parameters [7]. Zhiyong LI et la applied BP Neural network for prediction of machining accuracy of Aero-engine blade in ECM by taking the effect of voltage, feed rate, electrolyte concentration and initial gap [8]. Rao, RS et la investigated the optimal parameter setting and prediction for MRR in ECM using Taguchi based ANN for LM6Al/5\%SiC composite [9]. Abuzied H.H, et la developed ANN model for prediction of material removal rate and surface roughness in ECM process and other non-traditional machining process [10]. Fabricio J, et la showed the artificial neural networks for machining process surface roughness modeling [11]. SVM is a new machine learning method based on statistic learning theory and it is classified as one of computational approach developed by Vapnik [12]. Suykens and Vandenwalle have proposed the use of the LS-SVM for simplification of traditional of SVM [13]. Wang,P. et al applied LS-SVM for prediction model of surface roughness for grinding machining operation considering Linear, polynomial, Gauss RBF and sigmoid function as the kernel function[14]. Zhang et al applied SVM with multi-objective to develop a hybrid model for processing parameters optimization in micro-EDM using Gaussian function as a kernel function [15]. Sugumaran et al developed fault diagnostics of roller bearing using neighborhood score multiclass SVM for classification and decision tree for addressing the future selection process in EDM machining [16].

The present study is initiated to develop a multi input multi output MFNN model and LS-SVM models to predict the values of surface roughness \& material removal rate resulting from an electrochemical machining process and to evaluate optimum setting of surface roughness and material removal rate by using Taguchi method.

\section{EXPERIMENTAL WORKS}

\subsection{Experimental set up}

Experimental set up done for both calculation of MRR and Ra. The set up is shown in Fig. 1. Before machining we take the initial weight of work piece(EN19 tool steel) and after machining of 5minutes again we take the final weight. Initial weight and final weight gives the material removal rate per unit time when put in equation (2.1).

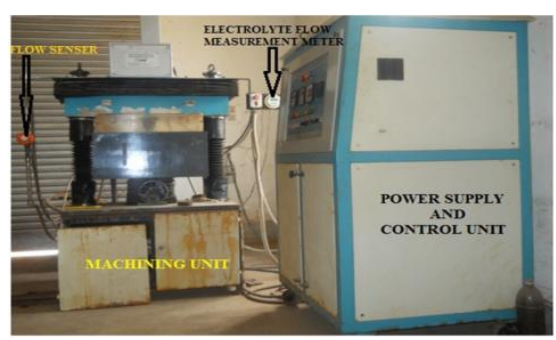

Fig. 1 Experimental setup

$$
\begin{gathered}
\text { MRR }=\frac{\left(W_{0}-W_{1}\right) \times 10^{-3} \times 10^{9}}{\rho_{w} \times t i m e} \text { in } \mathrm{mm}^{3} / \mathrm{min} \\
\text { where, } \\
\mathrm{W}_{0}=\text { initial weight in } \mathrm{Kg} \\
\mathrm{W}_{1}=\text { weight after one machining in } \mathrm{Kg} \\
\rho_{\mathrm{w}}=\text { density of material in } \mathrm{Kg} / \mathrm{m}^{3}
\end{gathered}
$$

And after end of each machining we have measure the surface roughness. Material removal based on anodic dissolution and the electrolyte flows between the electrodes and carries away the dissolved metal. In this process, a low voltage $(5-25 \mathrm{~V})$ is applied across two electrodes with a small gap size $(0.1 \mathrm{~mm}-0.5 \mathrm{~mm})$ and with a high current density around $2000 \mathrm{~A} / \mathrm{cm}^{2}$. Electrolytes are either acids or more generally basic salts dissolved in water. Typically $\mathrm{NaCl}$ dissolved with water is supplied to flow through the gap with a velocity of $20-30 \mathrm{~m} / \mathrm{s}$. Surface roughness is measured by Talysurf Profile meter. The work piece(EN19) material composition and mechanical properties are shown in Table 1 and Table 2 respectively.

Category: Steel

Class: Alloy steel 
Table 1 Material composition

\begin{tabular}{|l|l|}
\hline \multicolumn{1}{|c|}{ Element } & Weight \% \\
\hline $\mathrm{C}$ & $0.38-0.43$ \\
\hline $\mathrm{Mn}$ & $0.75-1.00$ \\
\hline $\mathrm{P}$ & 0.035 \\
\hline $\mathrm{S}$ & 0.04 \\
\hline $\mathrm{Si}$ & $0.15-0.3$ \\
\hline $\mathrm{Cr}$ & $0.8-1.10$ \\
\hline $\mathrm{Mo}$ & $0.15-0.25$ \\
\hline
\end{tabular}

Table 2 Mechanical properties

\begin{tabular}{|l|l|}
\hline Properties \\
\hline Density $\left(\mathrm{Kg} / \mathrm{m}^{3}\right)$ & $7.7 \times 10^{3}$ \\
\hline Poisson's ratio & $0.27-0.3$ \\
\hline Elastic Modulus(GPa) & $190-210$ \\
\hline Hardness(HB) & 197 \\
\hline
\end{tabular}

\subsection{Design of Experiment}

The experiment is designed according to Taguchi method. Three in put parameter, feed and voltage and flow rate of electrolyte are taken here. The level of each parameter is shown in Table-3. Process parameters are designed L18 orthogonal array as shown in Table 4. For this analysis we are considering data means not $\mathrm{S} / \mathrm{N}$ ratios.

Table 3 Level and magnitude of ECM parameters

\begin{tabular}{|c|l|l|l|l|}
\hline Factors & \multirow{2}{*}{ Unit } & \multicolumn{3}{|l|}{ Levels } \\
\cline { 3 - 5 } & & 1 & 2 & 3 \\
\hline Voltage(V) & Volt & 8 & 10 & 12 \\
\hline Feed rate(f) & $\begin{array}{l}\mathrm{mm} / \mathrm{mi} \\
\mathrm{n}\end{array}$ & 0.1 & 0.3 & 0.5 \\
\hline $\begin{array}{l}\text { Flow rate of } \\
\text { electrolyte }\end{array}$ & LPM & 10 & 15 & ----- \\
\hline
\end{tabular}

Table 4 Observation table based on L18 orthogonal array

\begin{tabular}{|l|l|l|l|l|}
\hline $\begin{array}{c}\text { Flow } \\
\text { Rate }\end{array}$ & Voltage & Feed & MRR & SR \\
\hline 10 & 8 & 0.1 & 14.2078 & 3.27 \\
\hline 10 & 8 & 0.3 & 29.6364 & 3.6 \\
\hline 10 & 8 & 0.5 & 38.8766 & 4.4 \\
\hline 10 & 10 & 0.1 & 21.5526 & 3.60 \\
\hline 10 & 10 & 0.3 & 33.7838 & 4.67 \\
\hline 10 & 10 & 0.5 & 40.7643 & 5.10 \\
\hline 10 & 12 & 0.1 & 35.3227 & 4.20 \\
\hline 10 & 12 & 0.3 & 38.8617 & 4.63 \\
\hline 10 & 12 & 0.5 & 48.4136 & 5.43 \\
\hline 15 & 8 & 0.1 & 26.9610 & 3.61 \\
\hline 15 & 8 & 0.3 & 36.5981 & 4.63 \\
\hline 15 & 8 & 0.5 & 46.1429 & 4.97 \\
\hline 15 & 10 & 0.1 & 30.0955 & 4.33 \\
\hline 15 & 10 & 0.3 & 39.2448 & 5.67 \\
\hline 15 & 10 & 0.5 & 52.7840 & 5.40 \\
\hline 15 & 12 & 0.1 & 39.4786 & 4.61 \\
\hline 15 & 12 & 0.3 & 45.8097 & 5.87 \\
\hline 15 & 12 & 0.5 & 64.5909 & 6.28 \\
\hline & & & & \\
\hline
\end{tabular}




\section{ANN(MFNN) Modeling}

The MFNN used here consists of three layers namely input layer, hidden layer and output layer as shown in Fig. 2. The Input layer of MFNN consists of different number of inputs variables according to the modeling of MFNN. The input variables are flow rate of electrolyte, feed rate and voltage. The number of output neuron is decided by the number of estimated parameters; therefore in this model two output neuron is taken corresponding to MRR and SR.

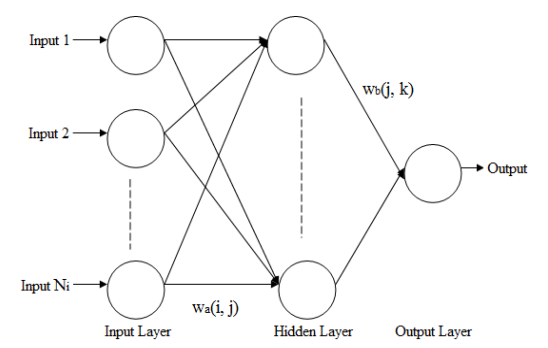

Fig. 2 Multilayer feed forward neural network

The Back Propagation Algorithm (BPA) is used to train the network. The sigmoid function represented by equation (3.1) is used as the activation function for all the neurons except for those in the input layer.

$\mathrm{S}(\mathrm{x})=1 /\left(1+\mathrm{e}^{-\mathrm{x}}\right)$

\subsection{Choice of Hidden Neurons}

The choice of optimal number of hidden neurons, $\mathrm{N}_{\mathrm{h}}$ is the most interesting and challenging aspect in designing the MFNN. There are various schools on thought in deciding the value of $N_{h}$. Simon Haykin [19] has specified that $\mathrm{N}_{\mathrm{h}}$ should lie between 2 and $\infty$. Hecht-Nielsen [20] uses ANN interpretation of Kolmogorov's theorem to arrive at the upper bound on the $\mathrm{N}_{\mathrm{h}}$ for a single hidden layer network as $2\left(\mathrm{~N}_{\mathrm{i}}+1\right)$, where $\mathrm{Ni}$ is the number of input neurons. However, this value should be decided very judiciously depending on the requirement of a problem. A large value of $\mathrm{N}_{\mathrm{h}}$ may reduce the training error associated with the MFNN, but at the cost of increasing the computational complexity and time. For example, if one gets a tolerably low value of training error with certain value of $\mathrm{N}_{h}$, there is no point in further increasing the value of $\mathrm{N}_{h}$ to enhance the performance of the MFNN. The input and the output data are normalized before being processed in the network as follows:

In this scheme of normalization, the maximum values of the input and output vector components:

$n_{i, \max }=\max \left(n_{i}(p)\right) p=1 \ldots N_{p}, i=1 \ldots N_{i}$

Where $N_{p}$ is the number of patterns in the training set and $N_{i}$ is the number of neurons in the input layer. Again,

$$
O_{k, \max }=\max \left(o_{k}(p)\right) p=1, \ldots . N_{p}, k=1, \ldots N_{k}
$$

Where, $N_{k}$ is the number of neurons in the output layer.

Normalized by these maximum values, the input and output variables are given as follows.

$n_{i, \text { nor }}(p)=\frac{n_{i}(p)}{n_{i, \max }}, p=1 \ldots . . N_{p}, \mathrm{i}=1, \ldots N_{i}$

and

$o_{k, n o r}(p)=\frac{o_{k}(p)}{o_{k, \max }}, p=1, \ldots N_{p}, i=1, \ldots N_{k}$

After normalization, the input and output variable lay [20] in the range of 0 to 1.

\subsection{Choice of ANN Parameters}

The learning rate, $\eta$ and the momentum factor, $\alpha$ have a very significant effect on the learning speed of the BPA. The BPA provides an approximation to the trajectory in the weight space computed by the method of steepest descent method [21]. If the value of $\eta$ is considered very small, this results in slow rate of learning, while if the value of $\eta$ is too large in order to speed up the rate of learning, the MFNN may become unstable (oscillatory). A simple method of increasing the rate of learning without making the MFNN unstable is by adding the momentum factor $\alpha$ [22]. The values of $\eta$ and $\alpha$ should lie between 0 and 1 [19].

\subsection{Weight Update Equations} follows:

The weights between the hidden layer and the output layer are updated based on the equation as

$w_{b}(j, k, m+1)=w_{b}(j, k, m)+\eta_{1} \times \delta_{k}(m) \times S_{b}(j)$ 
$+\alpha \times\left(w_{b}(j, k, m)-w_{b}(j, k, m-1)\right)$

Where $m$ is the number of iterations, $\mathrm{j}$ varies from 1 to $\mathrm{N}_{\mathrm{h}}$ and $\mathrm{k}$ varies from 1 to $\mathrm{N}_{\mathrm{k}} . \delta_{\mathrm{k}}(\mathrm{m})$ is the error for the $\mathrm{k}^{\text {th }}$ output at the $\mathrm{m}^{\text {th }}$ iteration. $\mathrm{S}_{\mathrm{b}}(\mathrm{j})$ is the output from the hidden layer[23].

Similarly, the weights between the hidden layer and the input layer are updated as follows:

$W_{a}(i, j, m+1)=w_{a}(i, j, m)+\eta_{1} \times \delta_{j}(m) \times S_{a}(j)$

$+\alpha \times\left(w_{a}(i, j, m)-w_{a}(i, j, m-1)\right)$

Where $\mathrm{i}$ varies from 1 to $\mathrm{N}_{\mathrm{i}}$ as there are $\mathrm{N}_{\mathrm{i}}$ inputs to the network, $\delta_{\mathrm{j}}(\mathrm{m})$ is the error for the $\mathrm{j}^{\text {th }}$ output after the $\mathrm{m}^{\text {th }}$ iteration and $\mathrm{S}_{\mathrm{a}}(\mathrm{i})$ is the output from the first layer. The $\delta_{\mathrm{k}}(\mathrm{m})$ in equation $(3.6)$ and $\delta_{\mathrm{j}}(\mathrm{m})$ in equation ( 3.7) are related as

$\delta_{j}(m)=\sum_{k=1}^{K} \delta_{k}(m) \times w_{b}(j, k, m)$

\subsection{Evaluation Criteria}

The Mean Square Error $\mathrm{E}_{\mathrm{tr}}$ for the training patterns after the $\mathrm{m}^{\text {th }}$ iteration is defined as:

$$
E_{t r}=\left(\sum_{p=1}^{P}\left(V_{b 1 p}-V_{b 2 p}(m)\right)^{2}\right) \times\left(\frac{1}{P}\right)
$$

Where $\mathrm{V}_{\mathrm{b} 1 \mathrm{p}}$ is the experimental values of MRR and SR, $P$ are the number of training patterns and $\mathrm{V}_{\mathrm{b} 2 \mathrm{p}}$ $(\mathrm{m})$ is the estimated values of MRR and SR after $\mathrm{m}^{\text {th }}$ iteration. The training is stopped when the least value of $E_{t r}$ has been obtained and this value does not change much with the number of iterations.

\section{LS-SVM Modeling}

The formulation of LS-SVM is introduced as follows. Consider a given training set $\left\{x_{i}, y_{i}\right\}, i=1,2, \ldots, N$, with input data $x_{i} \in R$ and output data $y_{i}=R$. The following regression model can be constructed by using non-linear mapping function $\phi\left({ }^{*}\right)[24]$.

$$
y=w^{T} \phi(x)+b
$$

Where $w$ is weight vector and $b$ is the bias term. As in SVM, it is necessary to minimize a cost function C containing a penalized regression error, as follows:

$$
\min C(w, e)=\frac{1}{2} w^{T} w+\frac{1}{2} \gamma \sum_{i=1}^{N} e_{i}^{2}
$$

subject to equality constraints

$$
y=w^{T} \phi\left(x_{i}\right)+b+e_{i}, i=1,2, \ldots ., N
$$

The first part of this cost function is a weight decay which is used to regularize weight sizes and penalize large weights. Due to this regularization, the weights converge to similar value. Large weights deteriorate the generalization ability of the LS-SVM because they can cause excessive variance. The second part of (4.2) is the regression error for all training data. The parameter $\gamma$, which has to be optimized by the user, gives the relative weight of this part as com-pared to the first part. The restriction supplied by (4.3) gives the definition of the regression error. To solve this optimization problem, Lagrange function is constructed as $L(w, b, e, \alpha)=\frac{1}{2}\|w\|^{2}+\gamma \sum_{i=1}^{N} e_{i}^{2}-\sum_{i=1}^{N} \alpha_{i}\left\{w^{T} \phi\left(x_{i}+b+e_{i}-y_{i}\right\}\right.$

where $\alpha_{i}$ are the Lagrange multipliers. The solution of (4.4) can be obtained by partially differentiating with respect to $w, b, e_{i}$ and $\alpha_{i}$

Then

$$
w=\sum_{i=1}^{N} \alpha_{i} \phi\left(x_{i}\right)=\sum_{i=1}^{N} \gamma e_{i} \phi\left(x_{i}\right)
$$

Where a positive definite Kernel is used as follows:

$$
K\left(x_{i}, x_{j}\right)=\phi\left(x_{i}\right)^{T} \phi\left(x_{j}\right)
$$

An important result of this approach is that the weights $(w)$ can be written as linear combination of the Lagrange multipliers with the corresponding data training $\left(x_{i}\right)$. Putting the result of (4.5) into (4.1), the following result is obtained as 
$y=\sum_{i=1}^{N} \alpha_{i} \phi\left(x_{i}\right)^{T} \phi(x)+b$

For a point $y_{i}$ to evaluate it is:

$y_{i}=\sum_{i=1}^{N} \alpha_{i} \phi\left(x_{i}\right)^{T} \phi\left(x_{j}\right)+b$

The vector follows from solving a set of linear equations:

$A\left[\begin{array}{l}\alpha \\ b\end{array}\right]=\left[\begin{array}{l}y \\ 0\end{array}\right]$

Where $A$ is a square matrix given by

$A=\left[\begin{array}{cc}K+\frac{I}{\gamma} & 1_{N} \\ 1_{N}^{T} & 0\end{array}\right]$

Where $\mathrm{K}$ denotes the kernel matrix with $i j$ th element in (4.5) and I denotes the identity matrix $\mathrm{N} \times \mathrm{N}, 1_{\mathrm{N}}=\left[\begin{array}{ll}1 & 1\end{array}\right.$ $1 \ldots . .1]^{\mathrm{T}}$. Hence the solution is given by:

$\left[\begin{array}{l}\alpha \\ b\end{array}\right]=A^{-1}\left[\begin{array}{l}y \\ 0\end{array}\right]$

All Lagrange multipliers (the support vectors) are non-zero, which means that all training objects contribute to the solution and it can be seen from (4.10) to (4.11). In contrast with standard SVM the LS-SVM solution is usually not sparse. However, by pruning and reduction techniques a sparse solution can easily achieved.

Depending on the number of training data set, either an iterative solver such as conjugate gradients methods (for large data set) can be used or direct solvers, in both cases with numerically reliable methods.

In application involving nonlinear regression it is not enough to change the inner product of $\left\langle\phi\left(x_{i}\right), \phi\left(x_{j}\right)\right\rangle$

(4.7) by a kernel function and the $i j$ th element of matrix $K$ equals to (4.5).

This show to the following nonlinear regression function:

$y=\sum_{i=1}^{N} \alpha_{i} K\left(x_{i}, x\right)+b$

For a point $x_{j}$ to be evaluated, it is:

$y_{j}=\sum_{i}^{N} \alpha_{i} K\left(x_{i}, x_{j}\right)+b$

For LS-SVM, there are lot of kernel function (radial basis function, linear polynomial), sigmoid, bspline, spline, etc. However, the kernel function more used is a simple Gaussian function, polynomial function and RBF. They are defined by:

$K\left(x_{i}, x_{j}\right)=\exp \left(-\frac{\left\|x_{i}-x_{j}\right\|^{2}}{\sigma_{s v}^{2}}\right)$
$K\left(x_{i}, x_{j}\right)=\left(x_{i}^{T} x_{j}+t\right)^{d}$

Where $d$ is the polynomial degree and $\sigma_{s v}^{2}$ is the squared variance of the Gaussian function, to obtained support vector it should be optimized by user. For $\alpha$ of the RBF kernel and $d$ of the polynomial kernel, in order to achieve a good generalization model it is very important to do a careful model selection of the tuning parameters, in combination with the regularization constant $\gamma$.

\subsection{Evaluation Criterion}

For the training patterns after the $m$ th iteration the mean square error $E_{t r}$ is defined as $E_{t r}=\left(\sum_{p=1}^{P}\left(V_{b 1 p}-V_{b 2 p}(m)\right)^{2}\right) \times\left(\frac{1}{P}\right)$ 

analysis of Electrochemical machining process where $V_{b 1}$ is denote the experimentally value of MRR for training purpose and same notation is used for SR for same purpose, $P$ is the number of training patterns and $V_{b 2 p}(m)$ is denote the modeled values of MRR and also SR after $m$ th iteration. Equation(4.16) used for estimation of $\mathrm{E}_{\mathrm{tr}}$ both for MRR and SR. When the least value of $E_{t r}$ has been obtained the training is stopped and this value does not change with number of iterations.

\section{RESULT AND DISCUSSION}

ANOVA and p main effect plots for MRR and SR are done by Taguchi technique using MINTAB 16. And with the help of 30 sets of experimental input-output patterns, the proposed modeling are carried out; 18 sets of input-output patterns used for training both networks and for testing purpose the remaining 12 sets are used. The software programs developed are used for implementation using MATLAB version 10.1.

\subsection{Optimal Parameter Setting and ANOVA}

The influences of various machining parameters on MRR are shown in Fig.3. The electrode feed rate has significant effect on MRR and it has directly proportional effect. MRR also increases with voltage and flow rate; however, according to the abridge ANOVA Table.5 the effect are less than the feed rate on MRR.

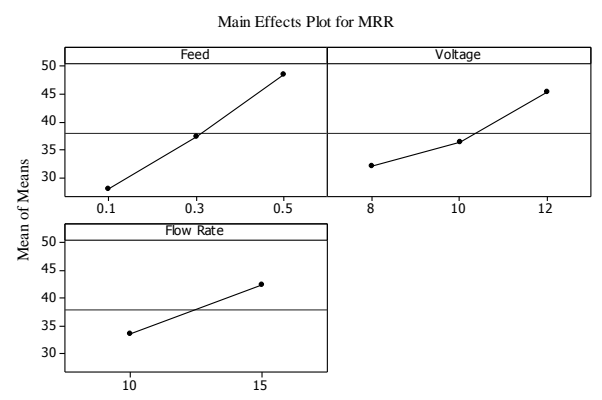

Fig. 3 Main effect plot for MRR

Table 5 ANOVA

\begin{tabular}{|c|c|c|c|c|c|c|}
\hline \multirow[b]{2}{*}{ Source } & \multicolumn{3}{|c|}{$\operatorname{MRR}\left(\mathrm{mm}^{3} / \mathrm{min}\right)$} & \multicolumn{3}{|c|}{$\mathrm{SR}(\mu \mathrm{m})$} \\
\hline & F & $\mathrm{P}$ & $\%$ contribution & $\mathrm{F}$ & $\mathrm{P}$ & $\begin{array}{l}\% \\
\text { contribution }\end{array}$ \\
\hline $\mathrm{f}$ & 146.86 & 0.000 & 55.44 & $\begin{array}{l}105.4 \\
6\end{array}$ & 0.000 & 45.28 \\
\hline $\mathrm{V}$ & 69.45 & 0.001 & 26.22 & 70.42 & 0.001 & 30.23 \\
\hline Flow Rate & 56.73 & 0.002 & 10.70 & 88.44 & 0.001 & 18.98 \\
\hline$f^{*} V$ & 5.53 & $0.063^{*}$ & 4.178 & 2.12 & $0.242 *$ & 1.82 \\
\hline f*Flow Rate & 3.89 & $0.115^{*}$ & 1.46 & 6.03 & $0.062 *$ & 2.59 \\
\hline \multirow[t]{2}{*}{ V*Flow Rate } & 3.24 & $0.145^{*}$ & 1.22 & 0.55 & $0.616^{*}$ & 0.24 \\
\hline & \multicolumn{3}{|c|}{$\begin{array}{l}S=1.605 \quad R^{2}=99.2 \% \\
R^{2}(a d j)=96.8 \%\end{array}$} & \multicolumn{3}{|c|}{$\begin{array}{l}S=0.1618 \quad R^{2}=99.1 \% \\
R^{2}(a d j)=96.4 \%\end{array}$} \\
\hline
\end{tabular}

The effect of various input parameters on SR are shown in Fig. 4. The graph shows that the feed rate, voltage and flow rate is directly proportional to SR. However the percentage of contribution are 45.28 30.23 and $18.98 \%$ for feed rate, voltage and flow rate respectively as shown in Table $5(*$ mark terms are insignificant).

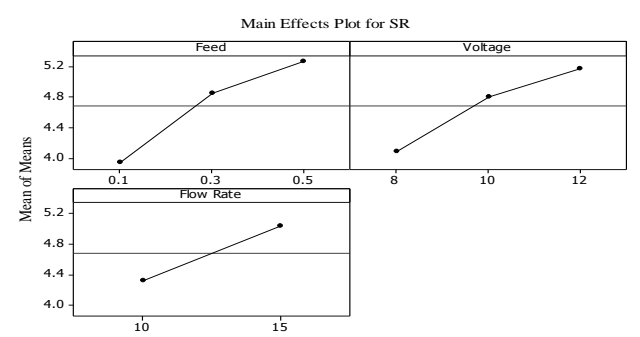

Fig. 4 Main effect plot for SR 


\subsection{Prediction of MRR and SR by MFNN}

From Tables 6-8 and Fig.5, it is quite obvious that when $\mathrm{N}_{\mathrm{h}}=10, \alpha=0.8$ and $\eta_{1}=0.9$, the MSE for training data is the lowest at $9.1098 \times 10^{-12}$. The sequential mode of training has been adopted. It may be noted that the range of the values of $\eta_{1}$ and $\alpha$ should be between 0 and 1 and value of $\mathrm{N}_{\mathrm{h}}$ should not more than 10 as per the Hecht-Nielsen criteria. Hence we have stopped at $\mathrm{N}_{\mathrm{h}}=10$ in Table 6 and in Table 7, stopped at $\eta_{1}=$ 0.9 . Table 8 shows that, stopped at $\alpha=0.8$.

Table 6 Variation of $\mathrm{E}_{\text {tr }}$ with $\mathrm{N}_{\mathrm{h}}\left(\eta_{1}=0.1, \alpha=0.6\right.$, iter $\left.=400\right)$

\begin{tabular}{|l|l|}
\hline $\mathrm{N}_{\mathrm{h}}$ & $\mathrm{E}_{\text {tr }}$ \\
\hline 1 & $5.6831 \times 10^{-6}$ \\
\hline 2 & $2.7895 \times 10^{-7}$ \\
\hline 3 & $1.9678 \times 10^{-7}$ \\
\hline 4 & $7.2582 \times 10^{-8}$ \\
\hline 5 & $6.4476 \times 10^{-8}$ \\
\hline 6 & $4.3436 \times 10^{-8}$ \\
\hline 7 & $2.3806 \times 10^{-8}$ \\
\hline 8 & $1.4038 \times 10^{-8}$ \\
\hline 9 & $1.145 \times 10^{-8}$ \\
\hline 10 & $1.0286 \times 10^{-8}$ \\
\hline
\end{tabular}

Table 7 variation of $\mathrm{E}_{\mathrm{tr}}$ with $\eta_{1}\left(\alpha=0.6\right.$, iter $\left.=400, \mathrm{~N}_{\mathrm{h}}=10\right)$

\begin{tabular}{|l|l|}
\hline$\eta_{1}$ & $\mathrm{E}_{\text {tr }}$ \\
\hline 0.4 & $5.5201 \times 10^{-9}$ \\
\hline 0.5 & $3.4123 \times 10^{-9}$ \\
\hline 0.6 & $2.3034 \times 10^{-9}$ \\
\hline 0.7 & $1.6515 \times 10^{-9}$ \\
\hline 0.8 & $1.2337 \times 10^{-9}$ \\
\hline 0.9 & $9.5846 \times 10^{-10}$ \\
\hline
\end{tabular}

Table 8 Variation of $\mathrm{E}_{\mathrm{tr}}$ with $\alpha\left(\eta_{1}=0.9\right.$, iter $\left.=400, \mathrm{~N}_{\mathrm{h}}=10\right)$

\begin{tabular}{|l|l|}
\hline$\alpha$ & $\mathrm{E}_{\text {tr }}$ \\
\hline 0.7 & $3.5209 \times 10^{-6}$ \\
\hline 0.8 & $9.1098 \times 10^{-12}$ \\
\hline 0.9 & $1.9798 \times 10^{-7}$ \\
\hline
\end{tabular}

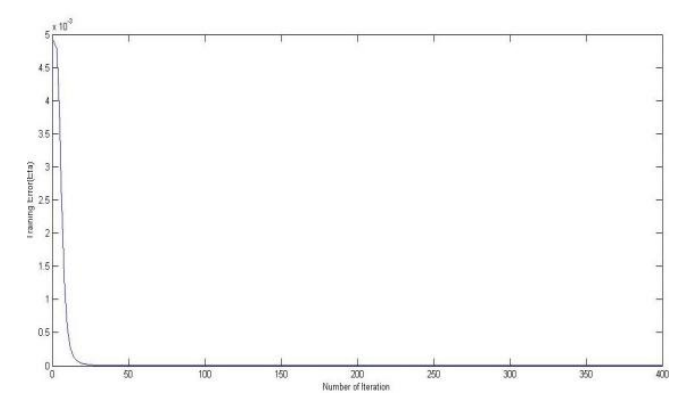

Fig. 5 Variation $\mathrm{E}_{\mathrm{tr}}$ with number of iterations $\left(\eta_{1}=0.9, \alpha=0.8\right.$ and $\left.\mathrm{N}_{\mathrm{h}}=10\right)$

\subsection{Prediction of MRR and SR by LS-SVM}

From Fig. 6 and Fig. 7, it is quite obvious that when $\sigma=0.31$, Sig2 $=5780.87$ and iteration $=400$, the data is well separated from LS-SVM hyper plane and mean square error (MSE) for training data is the lowest and equals to $4.55 \times 10^{-12}$. It may be noted that if the value of iteration is increased MSE will decrease. Finally, 
Taguchi integrated Least Square Support Vector Machine an alternative to Artificial Neural Network analysis of Electrochemical machining process for the MRR and SR, the test data are calculated by simply passing the input data in the LS-SVM and using $\alpha$ and $b$ for the kernel parameter. Here the conversion rate shows prediction of MRR and SR for Fig. 6-7.
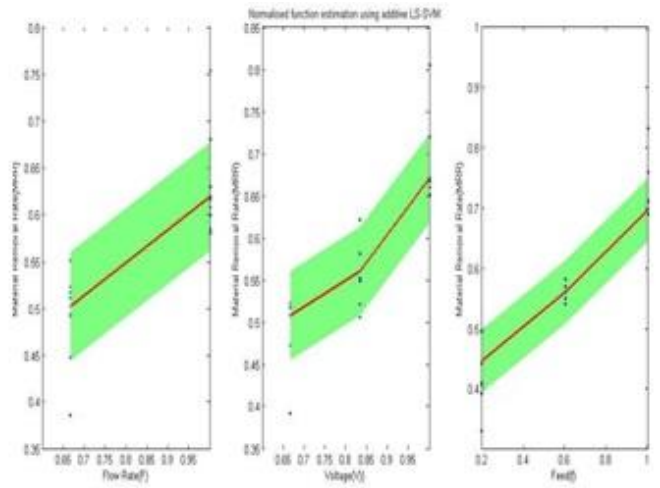

Fig. 6 shows change of conversion rate with respect to different input for training sample.
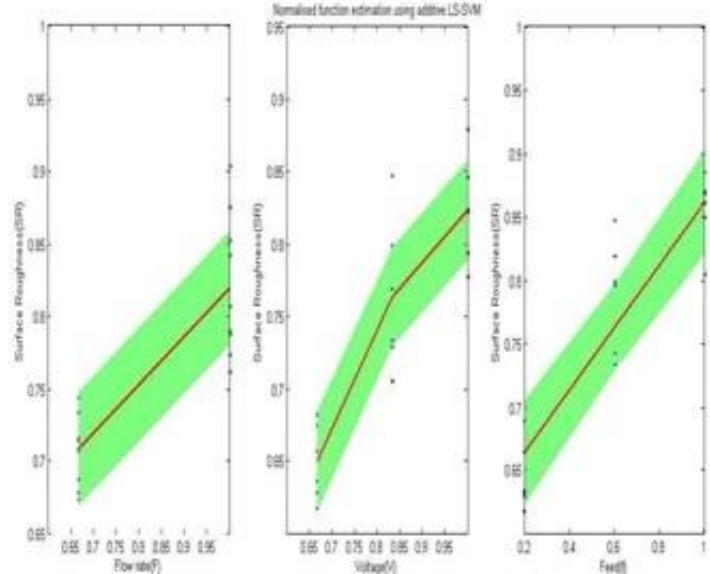

Fig. 7 shows change of conversion rate with respect to different input for training sample.

Table 9 MFNN and SVM Predicted table

\begin{tabular}{|c|c|c|c|c|c|c|c|c|c|c|}
\hline \multirow[b]{2}{*}{ 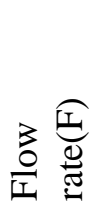 } & \multirow{2}{*}{ 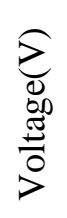 } & \multirow[b]{2}{*}{ 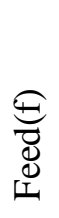 } & \multicolumn{3}{|c|}{ Surface roughness } & \multicolumn{3}{|c|}{ Material removal rate } & \multicolumn{2}{|c|}{$\begin{array}{l}\text { Testing Error for } \\
\text { SR }\end{array}$} \\
\hline & & & $\begin{array}{l}\text { Experi } \\
\text { mental } \\
\text { data } \\
\end{array}$ & $\begin{array}{l}\text { MFNN } \\
\text { predicted } \\
\text { data }\end{array}$ & $\begin{array}{l}\text { LSSVM } \\
\text { predict } \\
\text { ed data } \\
\end{array}$ & $\begin{array}{l}\text { Experim } \\
\text { ental } \\
\text { data }\end{array}$ & $\begin{array}{l}\text { MFNN } \\
\text { predicte } \\
\text { d data } \\
\end{array}$ & $\begin{array}{l}\text { LSSVM } \\
\text { predicte } \\
\text { d data }\end{array}$ & $\begin{array}{l}\text { MFNN } \\
\text { (MSE) }\end{array}$ & \begin{tabular}{|l} 
LSSVM \\
(MSE)
\end{tabular} \\
\hline 10 & 8 & 0.2 & 3.4334 & 3.4946 & 3.4581 & 24.6542 & 23.8462 & 25.2537 & \multirow{12}{*}{0.1258} & \multirow{12}{*}{0.0139} \\
\hline 10 & 8 & 0.4 & 4 & 4.4299 & 4.2087 & 36.2565 & 38.7694 & 32.4075 & & \\
\hline 10 & 10 & 0.2 & 4.1368 & 3.73327 & 4.1485 & 27.6682 & 26.8894 & 28.1439 & & \\
\hline 10 & 10 & 0.4 & 4.8833 & 5.03417 & 4.8991 & 37.2741 & 38.4375 & 35.2978 & & \\
\hline 10 & 12 & 0.2 & 4.415 & 4.25038 & 4.5125 & 35.0922 & 34.8755 & 34.2211 & & \\
\hline 10 & 12 & 0.4 & 5.0333 & 5.33033 & 5.2631 & 43.6376 & 42.9582 & 41.375 & & \\
\hline 15 & 8 & 0.2 & 4.119 & 3.72678 & 4.133 & 31.7795 & 30.2951 & 31.7088 & & \\
\hline 15 & 8 & 0.4 & 4.801 & 4.91906 & 4.8836 & 40.3705 & 42.5202 & 38.8626 & & \\
\hline 15 & 10 & 0.2 & 5 & 4.36185 & 4.8235 & 34.6701 & 32.8306 & 34.5991 & & \\
\hline 15 & 10 & 0.4 & 5.5333 & 5.31391 & 5.5741 & 47.0144 & 49.4476 & 46.7529 & & \\
\hline 15 & 12 & 0.2 & 5.23 & 4.59118 & 5.1874 & 39.6441 & 37.4207 & 40.6762 & & \\
\hline 15 & 12 & 0.4 & 6.073 & 5.87051 & 5.938 & 48.2003 & 51.6333 & 47.8301 & & \\
\hline
\end{tabular}




\subsection{Result Obtained}

Table 9 depicts the MSE obtained from two different models using different techniques. It may seen that the modeled values of MRR and SR for both the model closely follow the experimental values. And MSE values for both the models are 0.1258(for MFNN) and 0.0139(for LS-SVM). Hence it shows that LS-SVM model is better than MFNN model.

\section{CONCLUSION}

From the Taguchi analysis the most significant parameter towards the responses was found to be feed rate. The modeling of the ECM processes was successfully implemented. From model study, a LS-SVM with RBF Kernel and feed forward neural network using Back Propagation algorithm was designed, trainedand tested for conversion prediction of material removal rate and surface roughness. The selected neural network model which consists of one hidden layers with maximum 10 neurons in the hidden layers and LS-SVM with RBF kernel, successfully exhibited a good prediction capability with a low MSE for training and testing data. The ECM process yield can be computed by introducing the three independent variables including flow rate of electrolyte, feed rate, and voltage in the model. High conversion yield can be predicted and achievedby applying the neural network and least square support vector machine approaches which helps to avoid performing a large number of trial and error experiments to obtain the same results. LS-SVM gives the better prediction than ANN, this can lead to reduce the time and cost of the process and improve the quality of the product.

\section{REFERENCES}

[1] Muttamara A, Purktong S, "Improving the quality of groove in Electro chemical machining(ECM) processed by Taguchi method", International conference on Mechanical Engineering.20-22Oct.Ubon Ratchathani,2010.

[2] Dubey, A. K., Shan, H. S., Jain, N. K., "Analysis of surface roughness and out-of-roundness in electro-chemical honing process", International Journal Advance Manufacturing Technol, Proc lst Int and 22nd AIMTDR, IIT Roorkee (India) DOI 10.1007/s00170-007-1180-z Dec $21-23,2006$

[3] Singh S., Shan HS., Kumar P., "Parametric optimization of magnetic-field assisted abrasive flow machining by the Taguchi method", Qual Rel Eng Int 18:273 - 283 DOI 10.1002/qre.470, 2002.

[4] Asokan, A., R. Ravi Kumar, Jeyapaul, R., Santhi, M., "Development of multi-objective optimization models for electrochemical machining process", The International Journal of Advanced Manufacturing Technology, Volume 39, Numbers 1-2 / October, 2008.

[5] Tsai, K.M., Wang, P.J., "Predictions on Surface Finish in Electrical Discharge Machining based upon Neural Network Models", International Journal of Machine Tools \& Manufacture 41, 1385-1403, 2001.

[6] Shang, G.Q., Sun, C.H., “Application of BP neural Network For Predicting Anode Accuracy in ECM”, International Symposium on Information Science and Engineering, 2008.

[7] Kozak,J., Dabrowski, L., Lubkowski, K., Rozenek, M., Slawiński, R., "CAE-ECM system for electrochemical technology of parts and tools", Journal of Materials Processing Technology 107, 2000, p.293-299.

[8] Zhiyong, LI., Hua, JI., "Machining accuracy prediction of Aero-engine Blade in electrochemical Machining based on BP Neural network", International Workshop on information security and application(IWISA), November 21-22, 2009, pp 244-247.

[9] Rama Rao, S., Padmanabha, G., “Application of Taguchi methods and ANOVA in optimization of process parameters for metal removal rate in electrochemical machining of $\mathrm{Al} / 5 \% \mathrm{SiC}$ composites", International Journal of Engineering Research and Applications(IJERA), vol.2, Issue 3, pp192-197, 2012.

[10] Abuzied, HH., Awad Mohamed, A., Senbel, H.A., "Prediction of electrochemical machining process parameters using Artificial Neural Network", International Journal on Computer science and Engineering(IJCSE), vol.4.No.01, pp125-132, 2012.

[11] Silva Messias, B., Paiva, A.P., "Artificial neural networks for machining process surface roughness modelling”, International Journal Advance Manufacturing Technol, 49:879-902, 2010.

[12] Vapnik, V., The Nature of Statistical Learning Theory. ( Springer, New York. 1995).

[13] J.A.K Suykens, T Van Gestel, J De Brabanter, B De Moor, J Vandewalle, Least Square SVM. Singapore: World Scientific, 2002.

[14] Wang,P., Meng,Q., Zhao,J., Li,J. and Wang,X. Prediction of Machine Tool Condition Using Support Vector Machine. Journal of Physics Conference 305. 2011.

[15] Zhang,L., Jia,Z., Wang,F., and Liu,W. A hybrid model using supporting vector machine and multi-objective genetic algorithm for processing parameters optimization in micro-EDM, International Journal Advanced Manufacturing Technology 51, 575-586, 2010.

[16] Sugumaran,V., Sabareesh,G.R., and Ramachandran,K-I, Fault diagnostics of roller bearing using kernel based neighbourhood score multi-class support vector machine. Expert Systems with Applications 34, 3090-3098, 2008.

[17] D Hanbay,. "An expert system based on least square support vector machines for diagnosis of the valvular heart disease", Expert Systems with Application, 36(4), part-1, 8368-8374.2009

[18] Z Sahli,, A.Mekhaldi, R.Boudissa, S.Boudrahem, "Prediction parameters of dimensioning of insulators under non-uniform contaminated condition by multiple regression analysis", Electric Power System Research, Elsevier 2011 in press

[19] Simon Haykin.: 'Neural networks a comprehensive foundation' (Prentice Hall International Inc, New Jersey, USA, 1999), pp. 161$175,270-300$

[20] Hecht-Nielsen R.: Neuro Computing,( Addison-Wesley Publishing Company, Inc, 1990).

[21] S.Ghosh and N. K Kishore, "Modeling PD inception voltage of Epoxy Resin post insulators using an adaptive neural network", IEEE Transaction. Dielectrics and Electrical Insulation, Vol. 6, No. 1, 1999, pp. 131-134

[22] Rumel hart D.E., Mcclelland J.L. (EDS.): 'Parallel distributed processing: explorations in the microstructure of cognition' (MIT Press, 1986, vol. 1).

[23] S.Mohanty, S. Ghosh: "Artificial neural networks modeling of breakdown voltage of solid insulating materials in the presence of void”, Institution of Engineering and Technology Science. Measurement and Technology, Vol. 4, Iss. 5, pp 278-288, 2010.

[24] B.Zegnini, A.H. Mahdjoubi and M.Belkheiri "“ A Least Squares Support Vector Machines (LS-SVM) Approach for Predicting Critical Flashover Voltage of Polluted Insulators," 2011 IEEE Conference on Electrical Insulation and Dielectric Phenomena, Mexico,pp.403-406, 16-19 0ct 2011. 